\title{
Conceptual Study of NNARX Method for Solar Radio Burst Prediction Model Development
}

\author{
Mohd Rizman Sultan Mohd, Juliana Johari, and Fazlina Ahmat Ruslan
}

\begin{abstract}
Dependencies for electromagnetic spectrum-based technologies have increased with the introduction of digital broadcasting and high-speed internet. The solar weather which specifically related to the sun's activities caused an impact toward the stability and the continuity of electromagnetic spectrum on earth with the strike of solar flare. With the use of low-frequencies radio spectrometers, the early detection of solar flare can be made with the appearance of solar radio bursts. With the prediction model developed for solar radio burst, the effect of it can be reduced with a precaution action. Artificial Neural Network (ANN) had been widely applied in various prediction model development. Since solar radio flux is a highly non-linear data, Neural Network Autoregressive Model with Exogenous Input (NNARX) is considered for the solar radio burst prediction model development. NNARX is a dynamic ANN which had been widely applied in solar radiation prediction model development. In this paper, previous study on the application of NNARX will be discussed to develop an architectural concept in order to prepare the NNARX for solar radio burst prediction. By the end of this study, framework for the prediction model development is established to prepare future work for solar radio burst prediction using NNARX.
\end{abstract}

Index Terms - Solar Radio Burst, ANN, NNARX

\section{INTRODUCTION}

$S_{\text {weing }}^{\text {o }}$ OLAR radio burst is a natural phenomena related to the solar weather. It is usually referred to the early sign of solar flare event [1]. Because of this, the development of solar radio spectrometers had been done with several features and associated technique of observation. Solar radio spectrometers aim to captures the low frequencies transmitted by the sun. These low frequencies are travelled directly from the sun to the earth through the ionosphere layer [2]. The frequency signal from the sun, which referred to the solar radio is emitted by the sun in constant value. In the event of solar flare, the solar radio captured by the spectrometer will increase. This increased solar radio is defined as a solar radio burst. In other words, the solar radio burst is an early signal of solar flare event which could harm our stability of electromagnetic spectrum.

This manuscript is submitted on $16^{\text {th }}$ November 2020 and accepted on $27^{\text {th }}$ March 2021.

Mohd Rizman Sultan Mohd, Juliana Johari and Fazlina Ahmat Ruslan are with the Faculty of Electrical Engineering, Universiti Teknologi MARA, 40450 Shah Alam, Selangor (e-mail: fazlina419@uitm.edu.my)

1985-5389/C 2021 The Authors. Published by UiTM Press. This is an open access article under the CC BY-NC-ND license (http://creativecommons.org/ licenses/by-nc-nd/4.0/).
Solar radio burst could bring disturbance to entire technologies which depend on it such as telecommunication, internet, Global Positioning System (GPS), satellite, electrical grid, oil and gas pipeline distribution, energy chamber and many more [3].

TABLE I. SOLAR ACTIVITIES AND IMPACT ON EARTH

\begin{tabular}{|c|c|}
\hline Solar Activities & Impacts \\
\hline Solar Flares & $\begin{array}{l}\text { - The impact is from the development } \\
\text { of photons from solar flare. } \\
\text { - Impact earth surface which directly } \\
\text { face the sun. }\end{array}$ \\
\hline $\begin{array}{c}\text { Coronal Mass } \\
\text { Ejections }\end{array}$ & $\begin{array}{l}\text { - Large cloud of plasma erupts from } \\
\text { the sun. } \\
\text { - The cloud propagates in the eruption } \\
\text { direction. } \\
\text { - Impact the earth surface if the } \\
\text { propagation of cloud is facing the } \\
\text { earth. }\end{array}$ \\
\hline $\begin{array}{l}\text { High-Speed } \\
\text { Solar Wind }\end{array}$ & $\begin{array}{l}\text { - Develop from the coronal holes. } \\
\text { - Impact earth when the coronal holes } \\
\text { develop closer to solar equator. }\end{array}$ \\
\hline $\begin{array}{c}\text { Solar Energetic } \\
\text { Particles }\end{array}$ & $\begin{array}{l}\text { - Highly charged particles. } \\
\text { - Shocks form release by coronal } \\
\text { mass ejections and solar flares } \\
\text { formation. } \\
\text { - Impact earth with thecharged } \\
\text { particles that flows in magnetic field } \\
\text { path direction between sun and } \\
\text { earth. }\end{array}$ \\
\hline
\end{tabular}

Source: https://nasa.gov

Table I above shows the solar activities and its impact to the earth. Based on previous study on solar weather, there is a chance of having more than one solar activity and also the impact is varied [4]. The possibility is also because the events is related to each other. For example, Coronal Mass Ejections (CMEs) is usually occurs after the solar flare event [5]. Since the detection is based on the solar radio spectrometer, the detection is focusing on the value of the frequency which sensed by the spectrometers. By applying ANN, the prediction of the frequencies could be achieved. ANN had been applied in many prediction model developments such as flood prediction [6], agriculture-based prediction [7], business model prediction [8], faulty diagnosis model [9] and many more. There are two sub-categories for ANN; Static ANN and Dynamic ANN [10]. 
This paper will review the application of NNARX; the dynamic ANN method focusing on solar weather to develop the conceptual framework for solar radio burst prediction model.

\section{SOLAR RADIO BURST}

Spectrograms defined as a representative of radio frequency emits by the solar flares. The detection of solar flare is detected by the appearance of the radio frequency from the suns. To captures the solar radio frequency, spectrometers are used [11]. Spectrometers are used to identify and capture the radio frequency released from the sun through solar activities. This solar activity is in unstable form and can be vary from time to time. Because of the atmospheric layer of earth, there is a delay between the shock waves created by the solar flares before it hits the earth atmosphere [12]. Solar flares generate shock waves which propagate in the interplanetary medium. With the absence of the electron density profile on the propagation path, the velocity could be estimated [13]. The effect of solar radio burst can be seen in disturbance in Global Positioning Satellite (GPS) signals and excessive noise produced that can harm the user of communications during the occurrences [14].

Solar radio burst emission is based on solar flares event. It can be lasting ranging from a few seconds up to several hours. The characteristics of solar radio burst vary with wavelength. It is important to have a solid solar radio monitoring medium which could be used as forecast tools in detecting the solar flares [15]. Solar flare is a diverse dynamic activity which associated with high magnetic energy release, particle acceleration, radiation burst and release of plasma particles into the space [16]. Most of solar activities is due to the variation in its magnetic field. Solar magnetic field is developed in the convection zone which its occurrence is based from a dynamo process due to solar rotation and convection [17-18]. Solar flare is associated with solar spots with high magnetic field, solar cycle and Coronal Mass Ejections (CMEs). CMEs is defined as propagation of plasma particles along with magnetic field lines [19]. Usually, the energy of radiation is pass through the corona in low energy level. When the solar flare occurs, a tremendous amount of energy is emitted and this will increase the radiation level of the sun [20]. This also had impact the acceleration of plasma particles along with the magnetic field line induced. Accelerated particle causes intense radiations in entire wavelengths. Inside solar flare, gas temperature reaches up to approximately 10 to 20 million degrees Kelvin and could be as high as 100 million degrees Kelvin [21]. Based on previous research, there are three stages of solar flare had been identified as shown in Table II below [22].

Based on the analysis, it is reported that the entire stage of solar flare event could take up a few seconds or continues for an hour period. There are two categories of solar flares; single loop and two-ribbon flares. Single loop flares defined as one magnetic loop described as a flux tube brightens in X-rays and its shape and position is static during the occurences. Tworibbon flares is larger than a compact flare and happens near the solar prominence, a loop of plasma confined between two magnetic field lines [23].
TABLE II. SOLAR FLARE STAGES

\begin{tabular}{cc}
\hline Stages & Descriptions \\
\hline Pre-cursor & $\begin{array}{c}\text { The released of magnetic energy } \\
\text { and emission of soft x-ray }\end{array}$ \\
\hline Impulsive & $\begin{array}{c}\text { Accelerations of protons and } \\
\text { electrons in the particle and } \\
\text { emission of radio waves, gamma- } \\
\text { ray and hard x-ray }\end{array}$ \\
\hline Decay & $\begin{array}{c}\text { Soft x-ray will be gradually built } \\
\text { up and decay }\end{array}$ \\
\hline
\end{tabular}

The earth depends on its magnetosphere and atmosphere layer to protect the livings from the potential damage related to space weather [24]. The closed magnetic field of the magnetosphere absorbed the incoming shockwave due to the changes in the solar wind and deflect as many as possible the ionized radiation flux from the sun. The atmosphere layer will then absorbs the flux which consists of ionizing and extreme ultraviolet and soft $\mathrm{x}$-ray photons that produced by the solar flares.

There are five known types of solar radio burst namely Type I, Type II, Type III, Type IV and Type V [25]. This type of solar radio burst can be seen as individual or a combination of more than one type. Table III below shows the summary of all types of solar radio burst.

\section{TABLE III. TYPES OF SOLAR RADIO BURST}

\begin{tabular}{|c|c|}
\hline Solar Burst & Descriptions \\
\hline Type I & $\begin{array}{l}\text { - } \quad \text { Non-flare conditions. } \\
\text { - Consists of noise storm in the range of } \\
100 \text { to } 400 \mathrm{MHz} \text { with an hour time-scale } \\
\text { variation. } \\
\text { - Associated with burst component in } \\
\text { drifting chains of } 10 \text { to } 20 \mathrm{MHz} \text {. }\end{array}$ \\
\hline Type II & 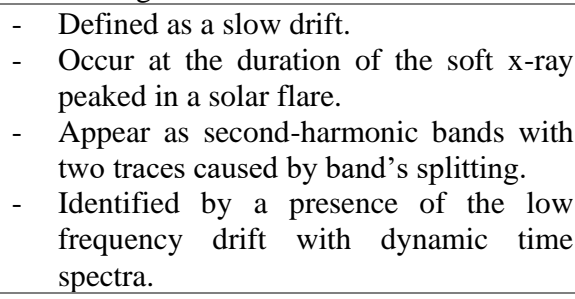 \\
\hline Type III & $\begin{array}{l}\text { - } \quad \text { Defined as a fast drift. } \\
\text { - } \text { Occur in duration of second. } \\
\text { - } \text { Occur in group of } 3 \text { and up to } 10 \text { with less } \\
\text { than } 60 \text { second duration. } \\
\text { - } \quad \text { Radio emission resulted from accelerated } \\
\text { electrons which propagate through } \\
\text { corona at high velocity. }\end{array}$ \\
\hline Type IV & $\begin{array}{l}\text { - Is an extension of continuum radiation } \\
\text { from Type I. } \\
\text { - Extend up to } 300 \mathrm{MHz} \text { frequency band } \\
\text { and often last for } 10 \text { to } 300 \text { minutes. }\end{array}$ \\
\hline Type V & $\begin{array}{l}\text { - Always associated with Type III with } \\
\text { duration less than } 60 \text { second. } \\
\text { - } \\
\text { Referred to an extension of Type III with } \\
\text { a flag-like image. } \\
\text { - Emission of Type V is equivalent to } \\
\text { quasi-continuous of the frequency } \\
\text { bandwidth of Type III. }\end{array}$ \\
\hline
\end{tabular}




\section{ARTIFICIAL NEURAL NETWORK}

ANN is an application of mathematical function to solve specific problems using the analogy of human brain as the decision-making process skill [26]. The architecture developed for the ANN is a simply the same with the structure of the biological brain. This architecture consists of numerous neurons which are interconnected in order and is placed in the dedicated group called layers. Based from the architecture analogy, there are three common layers of the ANN model which are, input layer, hidden layer and output layer.ANN is widely known as the conventional mathematical algorithms thatis used to solve a problem consisting a linear programming and logic-based calculations. ANN is widely appliesin solving a problem related to pattern recognition and matching [27], clustering [28] and classification [29]. The first concept of ANN was developed bya neurobiologist called Frank Rosenblatt in 1957 with the introducing of the Mark I Perceptron [30]. The model describes that the connectivity between neurons improved through a supervised learning process which will eventually reduce the misfit between actual and expected output. The expected output comes from a training data set. The misfit is then back-propagated through the entire network and allows the weights of the connections to be updated. The misfit between the actual and expected output of the network represents necessary information in which for learning performance improvements.

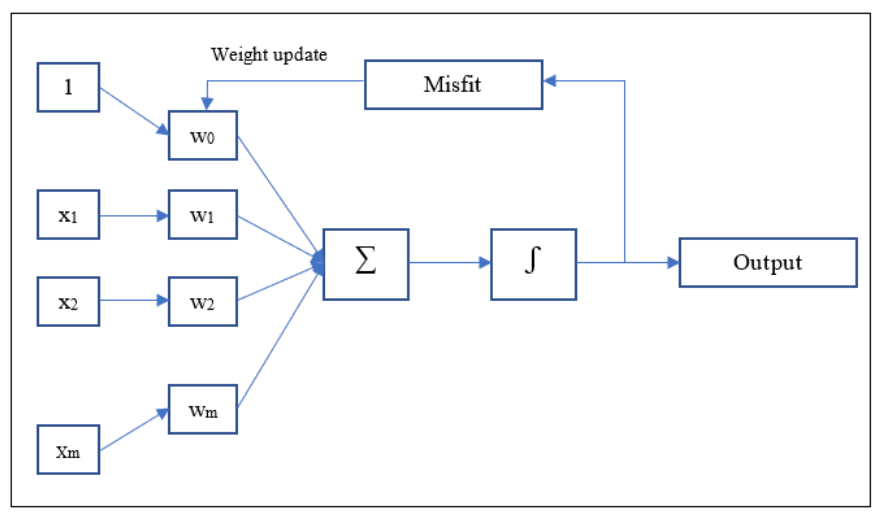

Fig. 1. The perceptron workflow.

Figure 1 shows the idea of the works of perceptron. At first, the perceptron receives the Inputs $x_{1}, x_{2}, \ldots, x_{m}$ [31]. The symbol ' 1 ' represents a bias in the Input Data. The combination of input with the Weights, w is used to compute the Net Input Function. The Net Input Function will be passed to the Activation Function that will produces a Binary Output of -1 or +1 in which corresponds to the predicted class label of the sample. Calculation of prediction's error is based on the output during the learning phase. In order to update the wights, the resultant value will be back-propagated. Eventually, the updated actions will continue to reduce the misfit between actual output and desired output.

Based from this fundamental study of perceptron to visualize the neural network methodology, further enhancement had been made and results in creating diverse architecture for ANN. To summarize, ANN can be divided into two main group; Static
ANN and Dynamic ANN. Static ANN is a non-looping network and dosen't have any feedback and delay mechanism in the connection. It also defined as a statistical regression tool because it deals with the non-linear function approximation sufficiently. The neural architecture of the Static ANN is shown in Fig. 2 below.

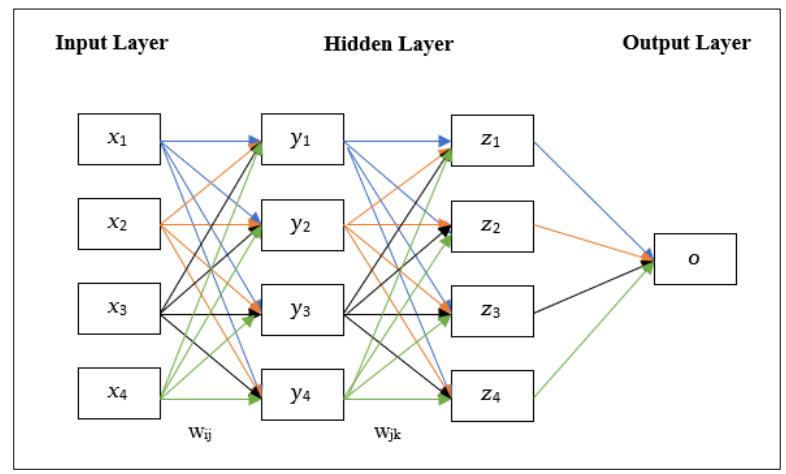

Fig. 2. The architecture of Static ANN.

Dynamic ANN is an updated version of Static ANN. Dynamic ANN has the capabilities of storing a memory. Because of this, Dynamic ANN can be trained to solve a sequential or time-varying patterns problems [32]. Dynamic ANN are trained with the same gradient-based algorithms of back-propagation as in Static ANN.Dynamic ANN delivers better performance as compared with Static ANN is because the gradient is computed in a more efficient way. Because of this complexity, the time consumed for a Dynamic ANN is high. Network training must be carried out multiple times to gets an optimal result [33].

\section{NNARX}

NNARX is the Dynamic ANN method [34]. In dynamic network, the output depends on various parameters such as current input, previous inputs, previous outputs and states of the network. NNARX has a parametric component with addition of a non-linear part. The single hidden layer of feed-forward ANN cater this non-linear approximation. In other words, NNARX is a current dynamic network consists of several layers network with a feedback connections. It also based on the linear ARX model which commonly applies in time-series modelling [35]. NNARX can be formulated as below:

$$
y^{\prime}(t)=F\left(y(t-1), y(t-2), \ldots y\left(t-n_{y}\right), x(t-1), x(t-2), \ldots, x\left(t-n_{x}\right)\right)
$$

From equation (1), the future value of the dependent output signal $y^{\prime}(t)$ is predicted based on previous output values with previous independent input values. There are two architecture of NNARX; parallel and series-parallel. Parallel architecture of NNARX can be formulated as equation (2) and series-parallel architecture of NNARX can be formulated as equation (3) below:

$y^{\prime}(t+1)=F\left(y(t), y(t-1), \ldots, y\left(t-n_{y}\right), x(t+1), x(t), x(t-1), \ldots, x\left(t-n_{x}\right)\right)$ 
$y^{\prime}(t+1)=F\left(y^{\prime}(t), y^{\prime}(t-1), \ldots, y^{\prime}\left(t-n_{y}\right), x(t+1), x(t), x(t-1), \ldots, x\left(t-n_{x}\right)\right)$

Where the function, $F$ defined as the mapping function of the NNARX with $y^{\prime}(t+1)$ as the output at specific time, $t+1$. The true past values of NNARX denoted as $y^{\prime}(t), y^{\prime}(t-1), \ldots, y^{\prime}\left(t-n_{y}\right)$ and also defined as the past output of NNARX while the input of the NNARX is denoted as $x(t+1), x(t), \ldots, x\left(t-n_{y}\right)$. The number of input delays is denoted as $n_{x}$ while number of output delay is denoted as $n_{y}$.

Parallel architecture of NNARX is called close-loop architecture where the output signal is feedback as the input of the feed-forwardnetwork asshown in Fig. 3. below.

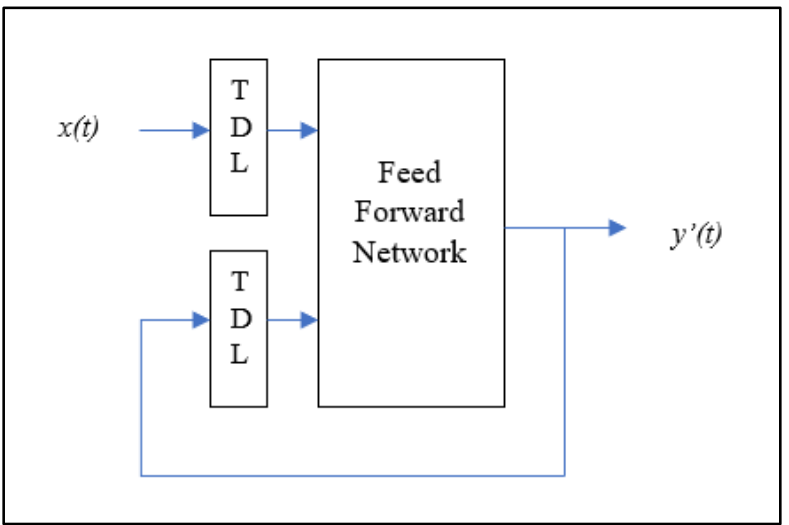

Fig. 3. Parallel architecture of NNARX.

The prediction is performed by the combination of present and previous input of $x(t)$ with additional feedback from past predicted values in form of time series $y^{\prime}(t)$.

The series-parallel architecture of NNARX is defined as open-loop architecture where it can consists of the true output that had been created during the training [36]. This would made true output is used without the needs of estimated output feedbackas shown in Fig. 4. The advantage of the series-parallel architecture is that the input is accurately feed in the network and resulting a purely feed-forward network architecture with the static back-propagation used as a training method.

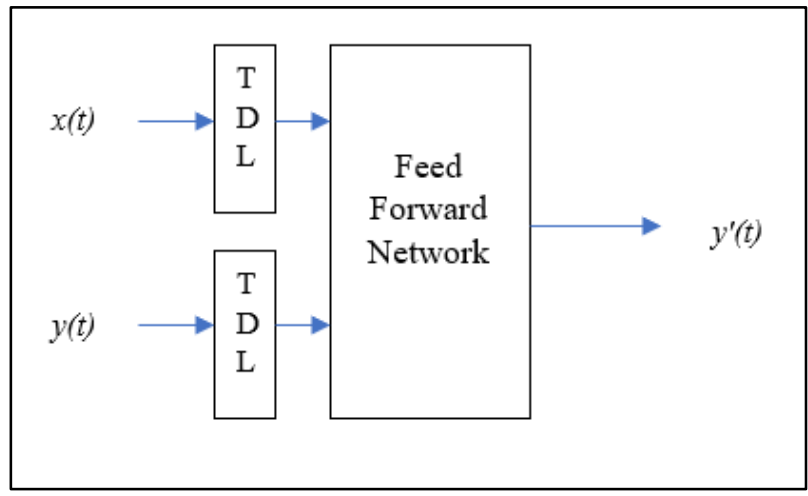

Fig. 4. Series-Parallel architecture of NNARX.
The future value predictionof the time series of $y(t-1)$ is performed with the combination of past and present value of $x(t)$ with additional feedback from past values in form of time series $y(t)$.

\section{APPLICATION OF NNARX IN SOLAR RADIATION PREDICTION}

According to general methodology of ANN as shown in Fig. 5 below, the data loaded into the model in database format and undergo data normalization process before it proceed with the Neural Network process. Data normalization will transform the data in the range of $[0,1]$. This to ensure the data is within the small-scale limit to avoid massive calculation perfomed and reduce the complexity by focusing on the training process. The method of NNARX starts with the learning and generalization of the system. Neural Network in general operates purposely on learning basis compared with a conventional programming method. The learning phase is where the Neural Network estimate the parameter to be feed in and study the behaviour and the types of parameter involved. The proportion of the input data is commonly on $40-40-20$ scale. This means, $40 \%$ of the data will used for training, $40 \%$ of the data used for testing and lastly $20 \%$ of remaining data used for the validation.

The inputs and outputs are totally depending on the prediction application. The network weight is constantly modified and adjusted during the training or learning phase. This weight adjustment is part of the specific learning algorithm process before it is tested in generalization phase. Generalization or testing phase will determine the Neural Network performance. It treats the output with non-learned input and the network capacity is degrades in the under-learning case.

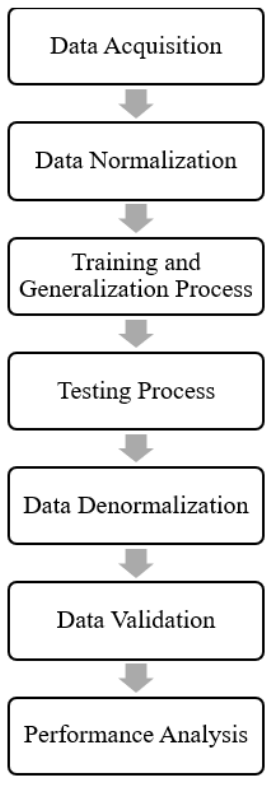

Fig. 5. Methodology flowchart for ANN. 
NNARX is a dynamic recurrent neural network. The concept of NNARX is generalization of non-linear parameter with the Autoregressive Model with Exogenous Input (ARX) which defined as the standard instrument linear black-box system identification [37]. The ability of tackling non-linear dynamic system had brought NNARX in applications of time-series prediction modelling [38]. To fully utilize this non-linear timeseries prediction, many utilizations had been made toward its memory-handling ability with the past values of predicted and true time series.

In order to have a solid prediction model using NNARX, there are many considerations made for several parameter that will be used as the input in the prediction model. Fig. 6 below shows the architecture of NNARX in solar radiation prediction model using Series-Parallel Architecture [39].

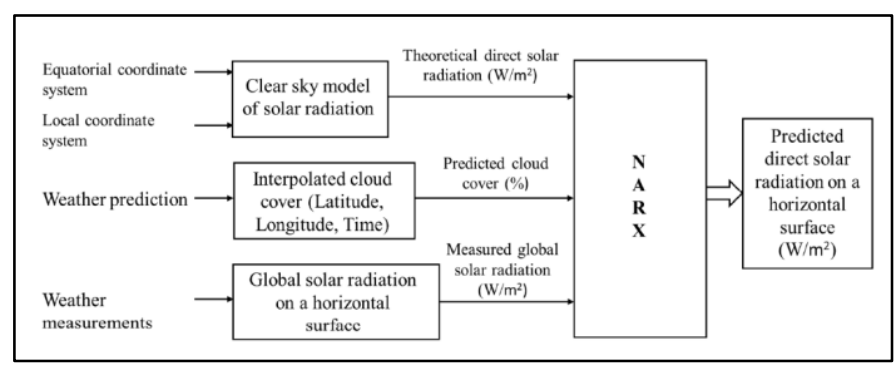

Fig. 6. The setup for NNARX prediction model for predicting direct solar radiation on a horizontal surface using series-parallel architecture.

In this study, the input is divided into two components; deterministic and statistical. Deterministic components are the mathematically description of direct solar radiation based on clear sky model and denoted as the endogenous input of NNARX. Statistical components concentrate on the cloud cover sky model and denoted as exogenous input of NNARX which involves an influential parameter of the direct solar radiation. Based from the results, this proposed method of NNARX had given a good indicator of performance based on the Mean Square Error (MSE) values as shown in Fig. 7 below.

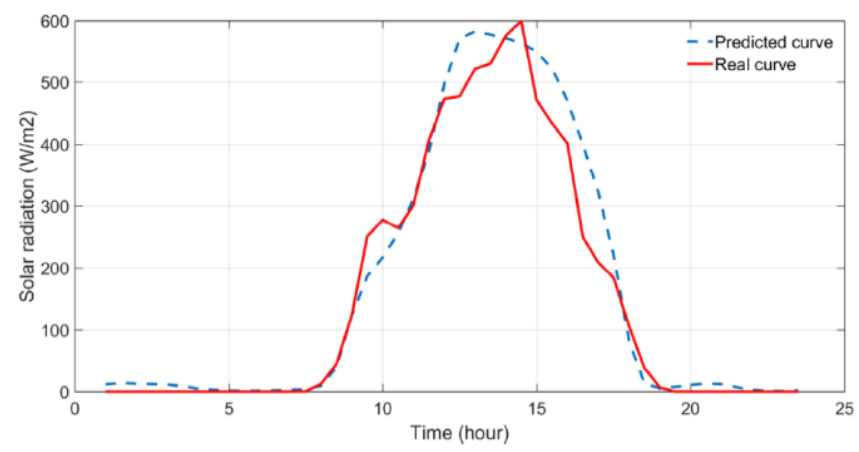

Fig. 7. The results obtained from the proposed method with the MSE value of 0.00279 .

Based from this study, it is shown that NNARX had intial data segmentation which not only used for the data tagging for training, testing and validation but also has features of segmentation the input data into statistical and deterministic parameter. This is due to the ability to handle exogenous input together with endogenous input in the Neural Network architecture. Solar radio burst prediction model development will consider the solar flare occurences, solar radiation value, sunspot occurences and the magnetic field readings using various space weather related instrumentations.

NNARX had been further applies in solar radiation prediction study because NNARX involves in parametric model which using different combination of weather prediction variables [40]. Based from this study, they utilized the parallel architecture and consists of the back-propagation algorithms method. Back-propagation method consists of training and recalled phase. During training phase, error of the network is calculated by means of gradient and the weights of output is adjusted [41]. Re-called phase runs with the finalization of the input variables and target combination There are two method of updating the weights; iteration method and calculation of mean values of input and output [42]. The new weight values and biases are calculated using equation (4) below:

$$
W_{i j, n}=U_{n}+\propto W_{i j, n}-1
$$

Where $W_{i j, n}$ is the current weight with a bias vectors, $\propto$ is the rate of momentum factor that determine the implication of past weights toward current value and $U_{n}$ defined as an update function of the system. Based from the study, the most common equation solving algorithm is the Levenberg-Marquardt (LM) training algorithm [43]. Based from LM, the update function of $U_{n}$ can be calculated using equation (5) below:

$$
U_{n}=-\left(J^{T} \times J+\mu I\right)^{-1} \times J^{T} \times e
$$

Where $J$ is the Jacobian matrix which contains the first derivatives of the network error based on weights and biases, $e$ is a vector of network error, $\mu$ is a number of scalar and $I$ refer to an identity matrix.

The training is done to study the lowest possible MSE values. The accuracy of the estimation based on the lowest MSE measured. Mean Bias Error (MBE) is used to indicate the average deviation of the predicted and measured value as shown in equation (6) below. The lower value of MBE, the better prediction can be made for a long-term model prediction.

$$
M B E=\frac{1}{N} \sum_{i=1}^{N}\left(I_{p, i}-I_{i}\right)
$$

MSE is calculated between actual output value and target output values. Network training can be stopped if the validation vectors saturated at certain MSE value. But in this study, further clarification had been made for the validation performance using the Regression Analysis. Regression analysis is done to determine the best-fit of the training, validation and test set. The perfect-fit can be determined if the data falls along a $45^{\circ}$ linear line between the outputs and target graph. Fig. 8 shows an example of linear regression analysis. 


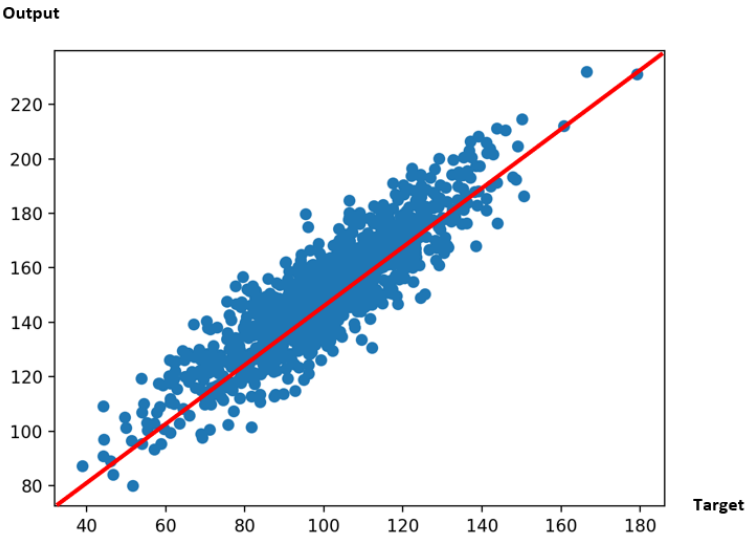

Fig. 8. Example of linear regression analysis graph indicate the output and target value.

The minimum value of MSE obtained from output and target value will be used as an indication of the number of neurons will be used in the NNARX architecture. This has further analysis by previous study made for static and dynamic neural network comparison [44].

To enhanced the prediction using NNARX, the introduction of other data processing had been implemented. This includes the application of image processing and other correctional method [45]. Based from the results in Fig. 9 below, it shows that the implementation of this hybrid approach had given better performance as indicated in the output produce.

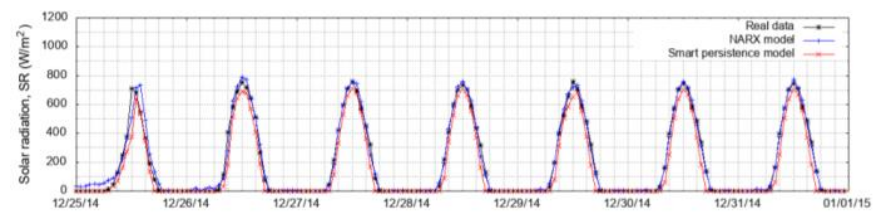

Fig. 9. The results obtained from the proposed hybrid method of NNARX in predicting solar radiation in Temixco, Mexico.

The performance analysis of NNARX model for prediction can be made using several parameter including MSE, Root Mean Square Error (RMSE),Mean Absolute Percentage error (MAPE) and MBE. This indication can be used to determine the difference made between the actual measured data and prediction-based time-series data.

\section{FINDINGS \& DISCUSSIONS}

Based from the conceptual study of NNARX, it had been observed that the application of NNARX had given a good prediction results on solar radiation. With the study, it is observed that NNARX had several abilities that suit the solar radio burst prediction model development. For the prediction model, the same methodology will be used. Fig. 10 below shows the proposed NNARX method in solar radio burst prediction considering the detail observed in the study.

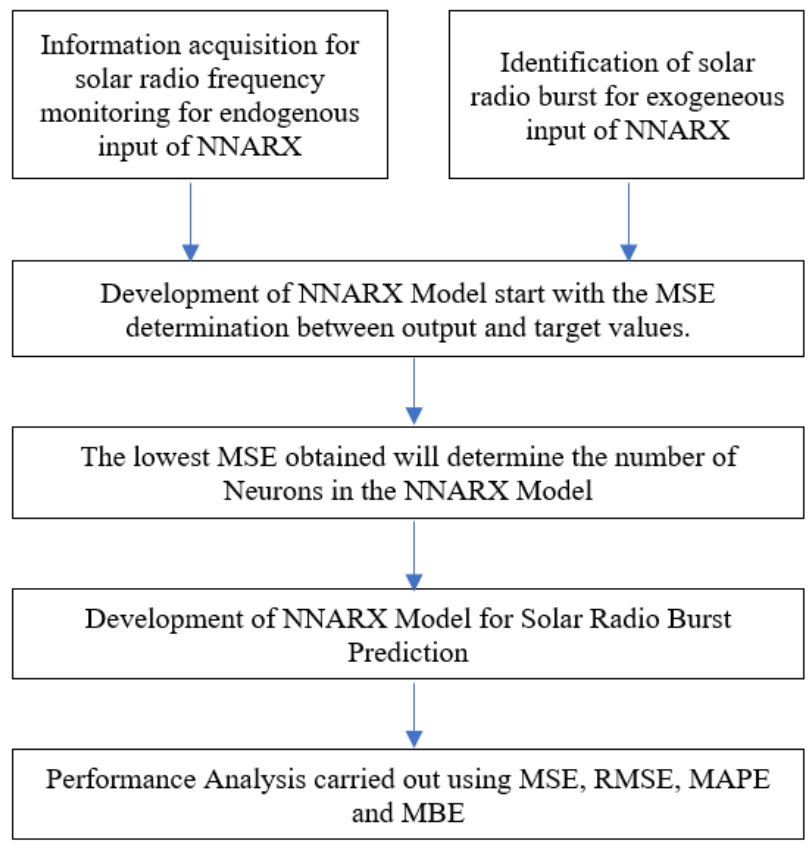

Fig. 10. Methodology best suit for the NNARX approach in solar radio burst prediction.

The ability to perform prediction on non-linear data had made NNARX is widely applied in the solar radiation prediction model development. With addition of exogenous input, NNARX can be used to deliver prediction results based on segmentation of deterministic and statistical component for the input parameter.Since solar radio burst has five different type, the prediction model must be trained with numerous data accordingly. The other related data for solar radio burst prediction will be going to the endogenous input while the classification of solar radio burst according to its type will be on the exogenous input as shown in Fig.10. There are five types of solar radio burst and it can be seen using solar radio spectrometers such as CALLISTO.

The aim of this study is to collect the information on how NNARX had been utilized in solar radiation prediction and what are the key information that will be used to develop solar radio burst prediction model using this ANN method.Based from the previous study, there are always a new things and certain missing link that need to be reconfigure in order to fully develop an efficient prediction model especially for non-linear data. NNARX had both endogenous and exogenous input parameter which had given us the complete information acquisition to the training part of the NNARX.Training part of the NNARX is used to determine the number of neurons for the prediction model. Training will be executed until the minimum MSE is obtained. This will be the guideline which had been setup for the behavior of the Neural Network in producing predicted output based on input parameter. 


\section{CONCLUSIONS}

This paper presents the review of the previous studies focusing on the NNARX application for solar radiation prediction model development. This is done because the application of NNARX for solar radio burst is a new approach. The similarity between solar radiation prediction and solar radio burst had made the conceptual study referred to the application of NNARX on solar radiation prediction. In the findings, it is observed that the NNARX had given the best prediction results for solar radiation because of its features. NNARX is a Dynamic Recurrent Neural Network that had the ability to deliver prediction on dynamic non-linear time-series data. NNARX also has the ability to segregate both deterministic and statistical input component which is an important features to ease the prediction based on a complex architecture. Based from the previous study, the input parameter is in time-series with hourly data acquisition. The prediction is made also in hourly basis. In solar radio flux measurement made by CALLISTO, the data also retrieved in hourly basis. In this paper, the study regarding to solar radiation is used to find the same research scope and limitation to get proper knowledge regarding to the application of NNARX toward solar radio burst. By the end of the conceptual study on NNARX, the proper methodology of prediction model development for solar radio burst is successfully obtained and will be a guideline for future work based on NNARX. It is also indicated that the hybrid approach of NNARX had been applied to enhanced the prediction results.

\section{ACKNOWLEDGMENT}

This work is supported by the Research Management Center Universiti Teknologi MARA Shah Alam under Sustainable Research Grant (Project Code: 600-RMC/SRC/5/3 (047/2020)). Authors would also like to thanks and acknowledge the Innovative Electromobility Research Laboratory (ITEM) and Faculty of Electrical Engineering Universiti Teknologi MARA Shah Alam for their support.

\section{REFERENCES}

[1] Z.S.Hamidi and N.N.M.Shariff, "The Analysis of Solar Radio Burst Type III Associated with Solar Activities Due to Magnetic Reconnection from 2012-2017,'Journal of Physics: Conference Series, Vol.1593, 2020.

[2] E.P. Carley, et.al, "Radio Observatiories and Instrumentation Used in Space Weather Science and Operations,"Journal of Space Weather \& Space Climate, Vol.10, No.7, 2020.

[3] A. Vourlidas, E.P. Carley and N. Vilmer, "Radio Obsevations of Coronal Mass Ejections: Space Weather Aspects," Frontiers in Astronomy and Space Sciences, Vol.7, No.43, 2020.

[4] K-L. Klein, C.S. Matamoros and P. Zucca, "Solar Radio Bursts as a Tool for Space Weather Forecasting,"ComptesRendus Physique, vol.19, pp36-42, 2018.

[5] M. R. S. Mohd, J. Johari and F. Ahmat, "A Review of Solar Radio Burst Detection Using CALLISTO," 2020 IEEE 10th International Conference on System Engineering and Technology (ICSET), Shah Alam, Malaysia, pp.61-66, 2020.

[6] M.A.S. Anuar, R. Z. A. Rahman, A. C . Soh, S. B. M. Noor, Z. D. Zulkafli, "A Promising Wavelet Decomposition-NNARX Model to Predict Flood: Application to Kelantan River Flood," International
Journal of Electrical and Computer Engineering Systems, Vol.11, No.2, 2020.

[7] A Escamilla-García, G.M. Soto-Zarazúa, M Toledano-Ayala, E. Rivas-Araiza and A. Gastélum-Barrios, "Applications of Artificial Neural Networks in Greenhouse Technology and Overview for Smart Agriculture Development,"Journal of Applied Science, 2020.

[8] J.C. Negrete, "Artificial Neural Networks in Mexican Agriculture, A Overview," International Journal of Research in Agriculture and Forestry, Vol.5, Issue.7, pp. 1-9, 2018.

[9] F. U. Coronado-Martinez, F. J. Ruiz-Sanchez and D. A. SuarezCerda, "Multi-NNARX Model of Complex Engineering Systems for Fault Detection and Diagnosis Applied to a Fossil Fuel Electric Power Plant,"IEEE International Autumn Meeting on Power, Electronics and Computing, 2017.

[10] Z. Wu, D. Rincon and P.D. Christofides, "Process Structure-based Recurrent Neural Network Modeling for Model Predictive Control of Nonlinear Processes," Journal of Process Control, Vol.89, pp7484, 2020.

[11] A.L. Lysenko, et.al, "X-ray and Gamma-ray Emission from Solar Flares," Physics, Vol.63, No.8, pp818-832, 2020.

[12] J.J. Curto, "Geomagnetic Solar Flare Effects: A Review," Journal of Space Weather and Space Climate, Vol.10, No.27, 2020.

[13] K. Bolmgren , C. Mitchell, J. Bruno and G. Bust, "Tomographic Imaging of Traveling Ionospheric Distrubances using GNSS and Geostationary Satellite Observations," Journal of Geophysical Research: Space Physics, Vol.125, 2020.

[14] Y.V. Yasyukevich, A.S. Yasyukevich and E.I. Astafyeva, "How Modernized and Strenghened GPS Signals Enhance the System Performance during Solar Radio Bursts," GPS Solutions, Vol.25, No.46, 2021.

[15] N.A.M. Norsham, et.al, "An Overview of Log Periodic Dipole Antenna (LPDA) UiTM Site and the Progress Status," Journal of Physics: Conference Series, Vol.1768, 2021.

[16] S. Khodairy, M. Sharaf, M. Awad, R. Abdel Hamed and M. Hussein, "Impact of Solar Activity on Low earth Orbiting Satellites," Journal of Physics: Conference Series, Vol.1523, 2020.

[17] Z. Xu, H. Ji, K. Ji , Y. Bi, B. Yang, J. Hong and J. Yang, "Localized Amplification of Magnetic Field in the Solar Photosphere Associated with a Rapid Moving Pore,"The Astrophysical Journal Letters, 2020.

[18] F. R. N. Schneider, S. T. Ohlmann, P. Podsiadlowski, F. K. Röpke, S. A. Balbus, R. Pakmor and V. Springel, , "Stellar Mergers as the Origin of Magnetic Massive Stars,"Nature, Vol.574, pp211-214, 2019.

[19] N.A.M. Norsham, Z.S. Hamidi and N.N.M. Shariff, "Detection of the Weak CMEs by CALLISTO System," Journal of Physics: Conference Series, Vol.1593, 2020.

[20] A. Kouloumvakos, et.al, "Evidence for a Coronal Shock Wave Origin for Relativistic Protons Producing Solar Gamma-Rays and Observed by Neutron Monitors at Earth," The Astrophysical Journal, Vol.893, No.1, 2020.

[21] N.M. Ansor, Z.S. Hamidi and N.N.M. Shariff, "The Implementation of Log Periodic Dipole Antenna (LPDA) and CALLISTO System on Solar Radio Bursts Observation," Journal of Physics: Conference Series, Vol.1768, 2020.

[22] G. Jerse, et.al. "Solar radio Diagnostic for Space weather with the Trieste Solar Radio System 2.0," 2020 IEEE International Conference on Wireless for Space and Extreme Environments, 2020.

[23] D.R. Jackson, et.al, "The Space weather Atmopshere Models and Indices (SWAMI) Project: Overview and First Results,"Journal of Space Weather and Space Climate, Vol.10, No.18, 2020.

[24] J.V. Wijesekera, "Analysis of Type II and Type III Solar Radio Burst," ${ }^{\text {th }}$ International Seminar of Aerospace Science and Technology, 2018.

[25] A.A. Kuznetsov, N. Chrysaphi, E.P. Kontar and G. Motorina, "Radio Echo in the Turbulent Corona and Simulations of Solar Driftpair Radio Bursts," The Atrophysical Journal, Vol.898, No.2, 2020.

[26] P. Wang, et.al, "Neurocognition-inspired Design with Machine Learning," International Journal of Design Sciences, Vol.6, 2020.

[27] I. Grabler, P.Scholle and H. Thiele, "Improving Scenario-Technique by a Semi-Automatized Consistency Assessment Based on Pattern Recognition by Artificial Neural Network," International Design Conference, 2020. 
[28] X.J. Luo, "A Novel Clustering-enhanced Adaptive Artificial Neural Network Model for Predicting Day-ahead Building Cooling Demand," Journal of Building Engineering, Vol.32, 2020.

[29] R. Bala and D. Kumar, "Classification Using ANN: A Review,"International Journal of Computational Intelligence Research, Vol.13, No.7, pp1181-1820, 2017.

[30] D.E. Babatunde, A. Anozie and J. Omoleye, "Artificial Neural Network and Its Applications in the Energy Sector - An Overview," International Journal of Energy Economics and Policy, Vol.10, No.2, pp250-264, 2020.

[31] L.Ling, C.Y.Mei, L.S. Qing and L.Lei, "Automatic Detection of Sunspots and Extraction of Their Feature Parameters," Chinese Astronomy and Astrophysics, Vol.44, Issue 4, pp462-473, 2020.

[32] P.P. Hadiyan, R. Moeini, and E. Ehsanzadeh, "Application of Static and Dynamic Artificial Neural Networks for Forecasting Inflow Discharges, Case Study: Sefidroud Dam Reservoir," Sustainable Computing: Informatics and Systmes, Vol.27, 2020.

[33] K. Posch and J. Pilz, "Correlated Parameters to Accurately Measure Uncertainty in Deep Neural Networks," IEEE Transactions on Neural Networks and Learning Systems, pp1-15. 2020.

[34] M. Islam, et.al, "An Overview of Neural Network,"American Journal of Neural Networks and Applications, Vol.5, No.1, pp7-11, 2019.

[35] A. Aljerme and H. Liu, "A Time-varying Neural-network-based ARX Model of the Traffic in Mobile Networks," $202010^{\text {th }}$ Annual Computing and Communication Workshop and Conference, 2020.

[36] M.R.S. Mohd, J.Johari and F.A. Ruslan, "Application of NNARX in Modeling A Solar Radiation," 2020 IEEE $8^{\text {th }}$ Conference on Systems, Process and Control, 2020.

[37] S. Naqvi, M. Ali, A. Z. Shaikh, Y. Iqbal, A. Rahim, S. Khadim and T. Altaf,, "Expoliting White Spaces for Karachi through Artificial Intelligence: Comparison of NARX and Cascade Feed Forward Back Propagation," International Journal of Advanced Computer Science and Applications, Vol.11, No.2, 2020.

[38] L.M.M. Amelot, U. S. Agathee, Y. Sunecher, "Time Series Modelling, NARX Neural Network and Hybrid KPCA-SVR Approach to Forecast the Foreign Exchange Market in Mauritius," African Journal of Economic and Management Studies, Vol.12, Issue 1, 2020

[39] Z. Boussaada, O. Curea, A. Remaci, H. Camblong and N. Mrabet Bellaaj, "A Nonlinear Autoregressive Exogenous (NARX) Neural Network Model for the Prediction of the Daily Direct Solar Radiation,"Energies, Vol.620, 2018.

[40] A. Choudhary, D. Pandey and S. Bhardwaj, "Global Solar Radiation Estimation Modeling Using Artificial Neural Network: A Case Study on Metro Cities of India," Intelligent Computing in Control and Communication, pp479-489, 2021.

[41] A. Choudhary, D. Pandeyand S. Bhardwaj, "Artificial Neural Networks Based Solar Radiation Estimation using Backpropagation Algorithm," International Journal of Renewable Energy Research, Vol.10, No.4, 2020.

[42] C.Chen, S. Reiz, C. Yu, H-J Bungartz and G. Biros, "Fast Approximation of the Gauss-Newton Hessian Matrix for the Multilayer Perceptron," SIAM Journal on Matrix Analysis and Applications, Vol.42, Issue 1, pp165-184, 2021.

[43] Yi-Chun Du and A. Stephanus, "Levenberg-Marquardt Neural Network Algorithm for Degree of Arteriovenous Fistula Stenosis Classification Using a Dula Optical Photoplethysmography Sensor,"Sensors, Vol.18, 2018.

[44] M. Alizamir, S.Kim, O.Kisi and M.Z. Kermani, "A Comparative Study of Several Machine Learning based on Non-linear Regression Methods in Estimating Solar Radiation: Case Studies of the USA and Turkey Regions," Energy, Vol.197, 2020.

[45] E. Rangel, E. Cadenas, R. Campos-Amezcua and J.L. Tena., "Enhanced Prediction of Solar Radiation Using NARX Models with Corrected Input Vectors," Energies, Vol.13, 2020.

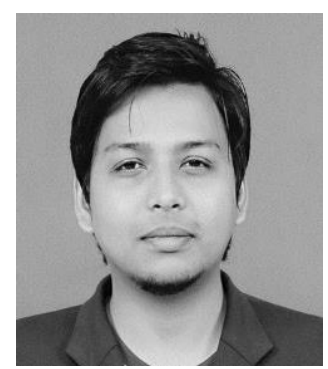

Mohd Rizman Sultan Mohd received Master degree in Electrical Engineering and Bachelor in Electronics Engineering from University Teknologi MARA. He is currently pursuing his Ph.D. at Universiti Teknologi MARA, Malaysia where he is currently developing solar energy prediction model using hybrid NNARX method. He is a Professional Engineer in Electronics Engineering and a Professional Technologist in Telecommunication and Broadcasting. $\mathrm{He}$ is currently working as Broadcasting Engineer at Jabatan Penyiaran Malaysia.

Juliana Johari received $\mathrm{PhD}$ in Micro-Engineering and NanoElectronics UniversitiKebangsaan Malaysia, Master degree in Biomedical Engineering from University of Surrey, United Kingdom and Bachelor in Electrical and Electronics Engineering from

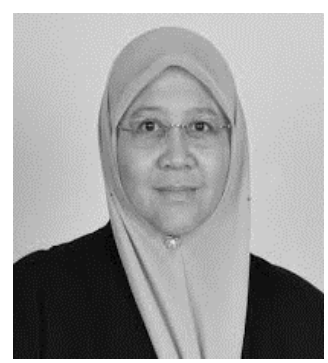
University of Strathclyde, United Kingdom. She is currently an Associate Professor in Control System and Instrumentation Engineering at the Faculty of Electrical Engineering, UniversitiTeknologi MARA Malaysia and Affliate Professor in Electronics and Instrumentation Engineering at the B.S. Abdur Rahman Crescent Institute of Science and Technology, Chennai, India. Area of specialization including robotics, automation, MEMS and Artificial Intelligence.

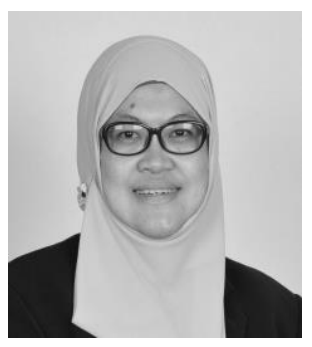

Fazlina Ahmat Ruslan received $\mathrm{PhD}$ in Electrical Engineering from UniversitiTeknologi MARA, Master degree in Microelectronics from UniversitiKebangsaan Malaysia and Bachelor in Electrical Engineering from UniversitiTeknologi MARA. She is currently working as Senior Lecturer in the Center of System Engineering Studies at Faculty of Electrical Engineering, Universiti Teknologi MARA Malaysia. Area of specialization including prediction system and Artificial Intelligence. 